\title{
Treatment Maneuvers for Benign Paroxysmal Positional Vertigo in the Very Old
}

\author{
Evangelos Anagnostou Evangelia Kararizou Ioannis Evdokimidis \\ Department of Neurology, University of Athens, Eginition Hospital, Athens, Greece
}

Dear Sirs,

Benign paroxysmal positional vertigo (BPPV) is the most common cause of vertigo and represents $1 \%$ of all patient visits to neurologists or ENT specialists [1]. The most frequent form of BPPV is canalolithiasis of the posterior semicircular canal with a lifetime prevalence of $2.4 \%$ [1]. The effect of treatment maneuvers has been extensively investigated in prospective studies with adequate patient samples and has been the subject of detailed systematic reviews and meta-analyses [2, 3]. Hence, both Epley and Semont maneuvers are considered reliable and safe therapies for BPPV. Aging contributes to fragmentation of otoconia and older adults often suffer from episodic dizziness due to BPPV [4] but systematic data on treatment options on older geriatric patients are lacking. Indeed, otherwise healthy but frail elders suffering from BPPV may miss the opportunity to benefit from therapeutic maneuvers, since physicians sometimes hesitate to apply appropriate Semont or Epley procedures in the elderly.

Here, we reviewed our patient charts between January 2009 and June 2016 looking for patients with posterior canal BPPV aged 85 years or older. We screened for cases with adequate documentation of (a) presenting symptoms, (b) positional nystagmus elicited by the side-lying or the

Table 1. Patients' demographics, applied maneuvers and outcome

\begin{tabular}{lllll}
\hline Subject's age & Gender & Maneuver & Times performed & Success \\
\hline 86 & f & Semont & 1 & Yes \\
89 & f & Semont & 2 & Yes \\
89 & f & Semont & d.n.a. & Yes \\
95 & m & Semont & 2 & Yes \\
91 & f & Semont & 3 & Yes \\
92 & f & Semont & 5 & No \\
92 & f & Semont & 1 & Yes \\
87 & m & Semont & d.n.a. & Yes \\
96 & f & Epley & 1 & Yes \\
88 & f & Epley & 3 & No \\
87 & m & Epley & 4 & Yes \\
90 & m & Epley & 1 & Yes \\
90 & f & Epley & d.n.a. & Yes \\
91 & m & Epley & 1 & Yes \\
\hline
\end{tabular}

d.n.a. = Data not available.

Dix-Hallpike maneuver and (c) result of the Semont or Epley maneuver.

This retrospective case series study was conducted in accordance with the Declaration of Helsinki and approved by our institution's ethics committee.

Fourteen eligible cases were identified, with ages ranging from 86 to 96 years (mean age 90.2 years; table 1$)$. Most patients were women $(n=9)$ and 9 cases were $\geq 90$ years. Eight patients received a side-lying and subsequently a Semont maneuver, while 6 patients underwent the Dix-Hallpike and the Epley repositioning procedure. Ages were similar in both groups (Semont: $90.1 \pm 2.9$ years, Epley: $90.3 \pm 3.1$ years) Many cases required repetitions of the treatment maneuvers, and BPPV failed to

\section{KARGER}

(C) 2016 S. Karger AG, Basel 
resolve in 1 subject in each group. Data on required maneuver repetitions were not available in 3 cases. Hence, Semont maneuver cleared the symptoms in 7 out of 8 patients and Epley maneuver in 5 out of 6. Formal statistical testing was not meaningful because of the smallness of the samples.

Overall, the above data indicate that success rates of Semont and Epley maneuvers in advanced old age are comparable to published studies on younger populations, which demonstrate symptom resolution in $75.9-95 \%$ of cases [5-7]. No side effects attributable to the maneuvers could

\section{References}

1 von Brevern M, Radtke A, Lezius F, et al: Epidemiology of benign paroxysmal positional vertigo: a population based study. J Neurol Neurosurg Psychiatr 2007;78:710-715.

2 Helminski JO, Zee DS, Janssen I, Hain TC Effectiveness of particle repositioning maneuvers in the treatment of benign paroxysmal positional vertigo: a systematic review. Phys Ther 2010;90:663-678. be retrieved from our charts, which can be regarded as an indication of a fair tolerance of the procedures. Interestingly, more than half of our subjects were $\geq 90$ years, and this subgroup showed no differences with respect to efficacy and safety of the maneuvers. It should be noted, however, that no patient in the present series appeared to suffer from any other serious medical condition, and therefore, our findings are not generalizable to the entire above 85 -year-old population. Regarding the 2 unsuccessful cases, no further details could be obtained, because we lost followup.

3 Hunt WT, Zimmermann EF, Hilton MP: Modifications of the Epley (canalith repositioning) manoeuvre for posterior canal benign paroxysmal positional vertigo (BPPV). Cochrane Database Syst Rev 2012; 4:CD008675.

4 Parham K, Kuchel GA: A geriatric perspective on benign paroxysmal positional vertigo. J Am Geriatr Soc 2016;64:378-385.

5 Yimtae K, Srirompotong S, Srirompotong S, Sae-Seaw P: A randomized trial of the canalith repositioning procedure. Laryngoscope 2003 ; 113:828-832.
Clearly, systematic and prospective data on larger elder samples using both Semont and Epley maneuvers are needed in order to draw safe conclusions regarding efficacy and tolerance. Nonetheless, the present case series suggest that therapeutic maneuvers are feasible, effective and safe and should not be withheld from patients in advanced old age.

\section{Disclosure Statement}

The authors state that there is no conflict of interest.
6 von Brevern M, Seelig T, Radtke A, TielWilck K, Neuhauser H, Lempert T: Shortterm efficacy of Epley's manoeuvre: a doubleblind randomised trial. J Neurol Neurosurg Psychiatry 2006;77:980-982.

7 Chen Y, Zhuang J, Zhang L, et al: Short-term efficacy of Semont maneuver for benign paroxysmal positional vertigo: a double-blind randomized trial. Otol Neurotol 2012;33:1127-1130. 